\title{
Dynamics and Control of a 4-dof Wearable Cable-driven Upper Arm Exoskeleton
}

\author{
Elizabeth A. Brackbill, Ying Mao, Sunil K. Agrawal, Madhu Annapragada, Venkatesh N. Dubey
}

\begin{abstract}
In this paper, we present the dynamics, control, and preliminary experiments on a wearable upper arm exoskeleton intended for human users with four degrees-of-freedom (dof), driven by six cables. The control of this cable-driven exoskeleton is complicated because the cables can transmit forces to the arm only under tension. The standard PD controllers or Computed torque controllers perform only moderately since the cables need to be in tension. Future efforts will seek to refine these control strategies and their implementations to improve functionality of a human user.
\end{abstract}

\section{INTRODUCTION}

$\mathrm{R}$ OBOTIC powered exoskeletons are being used increasingly today both as a tool for rehabilitation and/or assistance of patients with movement disabilities, as well as for augmentation of strength or agility in able bodied users. Lower extremity devices, such as Lokomat [1], BLEEX [2], and HAL [3] have been built for the lower extremity. They are constructed out of rigid members driven by actuators positioned at the joints. Many upper extremity exoskeleton designs also follow this design strategy ([4],[5],[6]). Some designs modify this strategy slightly, with motors positioned farther away from the joints and mounted on the base of the device to reduce the load on the moving limb ([7], [8]). Despite thoughtful design, they typically add weight along the extremities, making the limbs heavy and awkward to manipulate. Additionally, the robotic arm rehabilitation devices currently in use or being developed are large, some mounted to a wheelchair [7]. While a larger device is acceptable for a training activity, a wearable device is required for assistance in daily life activity.

Some devices have attempted to decrease the size and weight of the exoskeleton by replacing some or all of the rigid links with lightweight cables, so that they are not bulky and the dynamics are similar to those of a human arm without an exoskeleton. Although not itself an exoskeleton, Man et al present an arm manipulator with a hybrid drive mechanism, such that cables are used to control the elbow and wrist, while a modular serial mechanisms was used at the shoulder [9]. Taking it a step further, Yang et al propose a full $7 \mathrm{dof}$ arm exoskeleton driven entirely by cables [10]. Using a modular

Revised final Manuscript received on February 15, 2009.

E.A. Brackbill, Y. Mao, and S.K. Agrawal are with the Mechanincal Engineering Department, University of Delaware, Newark, DE 19702 USA, e-mail: \{ebrackb, maoying, agrawal\}@udel.edu. M. Aannapragada is with Automation Research Group in Newark, DE (USA) and V. Dubey is with the School of Design Engineering at the Bournemouth University in Bournemouth (England). design and redundant cables, they found favorable results of both displacement and tension analyses. These designs are quite promising, but were purely theoretical.

Our work seeks to design an upper extremity exoskeleton that is cable driven rather than being constructed out of rigid links. By taking advantage of the properties of cable driven designs, using lightweight cables, the entire system is capable of more agile dynamic movements than a comparable rigid design. Also, the actuators can be positioned on the torso of the wearer, so that the only added weight on the upper arm are those of the attachment cuffs and cables. Finally, the use of torso- mounted actuators can be combined with parallel gravity balancing [11], as previously demonstrated in both a lower [12] and upper extremity exoskeleton [13]. With these features, we expect this exoskeleton to be better suited as an assistance device for impaired or elderly wearers for activities of daily living (ADL).

In this paper, we present a description of our device, including the design, dynamics, optimization, and control structures. Finally, we present simulation and preliminary experiment results.

\section{EXOSKELETON DESIGN}

\section{A. Description}

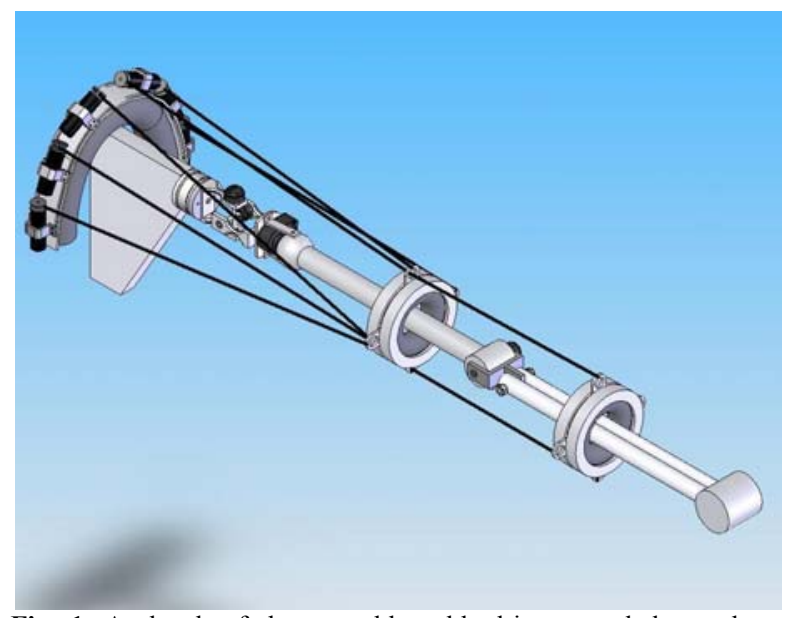

Fig. 1. A sketch of the wearable cable-driven exoskeleton shown attached to an anthropomorphic arm.

The cable driven arm exoskeleton is made up of three cuffs which attach to the user at the shoulder, mid- upper arm, and mid-forearm (Fig. 1). The cuffs are rigid, and can be adjusted using inflatable tubes to ensure a snug, yet comfortable, fit for the user. In the current prototype, all cuffs are made from aluminum. The shoulder cuff is a semi-circle to fit the 
shoulder. It has radial extensions for the attachment of the cables for the device. Both arm cuffs are circular, with small radial extensions for the cable attachment points. There are six cables which connect to the cuffs and are used to drive the device actively. All six originate at the shoulder cuff, where the motors are mounted. The attachment points at the shoulder are adjustable both radially and angularly to allow optimal positioning of the motors [15]. Four of the wires terminate at the bicep cuff, and are responsible for the three degrees-of-freedom at the shoulder (plane of elevation, angle of elevation, internal/external rotation). The remaining two cables pass through the bicep cuff, and terminate at the forearm cuff. These two cables are responsible for elbow flexion and extension.

The exoskeleton is controlled by six Maxon 30-Watt EC 45 flat brushless motors, mounted on the shoulder cuff. Each motor uses Maxon's DEC24/3 brushless motor amplifier. These are then powered by a modular ASTEC MP series power supply. The controllers were interfaced via a dSPACE DS1103 PPC Controller Board using ControlDesk user interface. The motors are equipped with Hall Sensors which provide feedback to the dSPACE board.

\section{B. Dynamics}

The dynamic equations of motion of the device were derived using Lagrangian method, and the calculations were done in Maple. Values for the masses, inertias, and lengths of the arm were estimated. The DH parameters for the system are listed in Table 1.

TABLE I

\begin{tabular}{ccccc}
\multicolumn{3}{c}{ DH PARAMETERS FOR ARM EXOSKELETON } \\
\hline \hline Link & $\mathrm{a}_{\mathrm{i}}$ & $\alpha_{\mathrm{i}}$ & $\mathrm{d}_{\mathrm{i}}$ & $\theta_{\mathrm{i}}$ \\
\hline 1 & 0 & 90 & 0 & $\theta_{1}$ \\
2 & 0 & 90 & 0 & $\theta_{2}$ \\
3 & 0 & 90 & $\mathrm{~d}_{3}$ & $\theta_{3}$ \\
4 & $\mathrm{a}_{4}$ & 0 & 0 & $\theta_{4}$ \\
\hline \hline
\end{tabular}

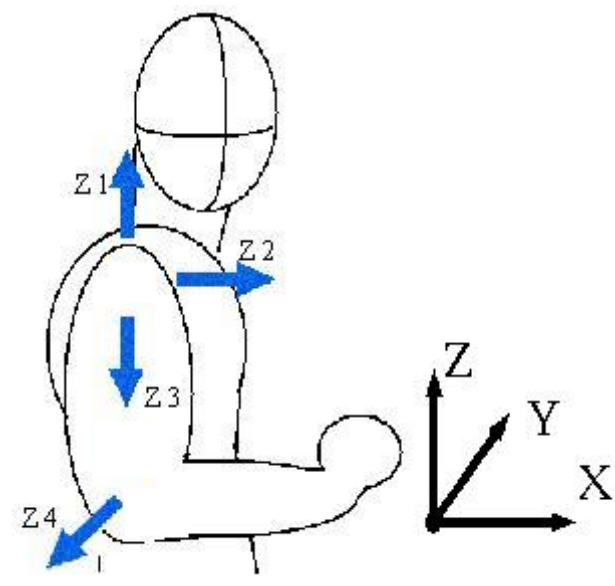

Fig. 2. Joint rotation axes and zero position. Angle $q_{1}$ is measured about $\mathrm{Z} 1, \mathrm{q}_{2}$ about $\mathrm{Z} 2$ etc.

The coordinates $\left(\mathrm{q}_{1}, \mathrm{q}_{2}, \mathrm{q}_{3}\right)$ represent the 3-dofs at the shoulder and can also be interpreted in terms of the standard Euler angles where $\mathrm{q}_{1}$ is plane of elevation, $\mathrm{q}_{2}$ is angle of elevation, and $\mathrm{q}_{3}$ is the internal/ external rotation [14]. The remaining angle $\mathrm{q}_{4}$ is the elbow flexion. The zero position of the arm is shown in Fig. 2 and is in accordance with the DH convention; the upper arm is at the side parallel to the torso, but the elbow is in anatomically flexed position, with the forearm pointed forward (positive $\mathrm{x}$ direction). The equation of motion of the system are given by:

$$
\mathrm{D}(\mathrm{q}) \ddot{\mathrm{q}}+\mathrm{C}(\mathrm{q}, \dot{\mathrm{q}}) \dot{\mathrm{q}}+\mathrm{g}(\mathrm{q})=\mathrm{J}(\mathrm{q})^{\mathrm{T}} \mathrm{T}(\mathrm{t})
$$

where $\mathrm{q}=\left(\mathrm{q}_{1}, \mathrm{q}_{2}, \mathrm{q}_{3}, \mathrm{q}_{4}\right)^{\mathrm{T}}$ are the generalized coordinates, $\mathrm{D}(\mathrm{q})$ is the $(4 \times 4)$ inertia matrix, $C(q, \dot{q})$ is the vector of nonlinear centripetal terms, $g(q)$ is the vector of gravity terms, $J(q)$ is the Jacobian relating the cable tensions to joint torques, and $\mathrm{T}(\mathrm{t})$ is a six dimensional cable tension vector. As expected, the dynamic equations are fairly non-linear and complex, hence the detailed equations are not included in this paper.

\section{Optimization}

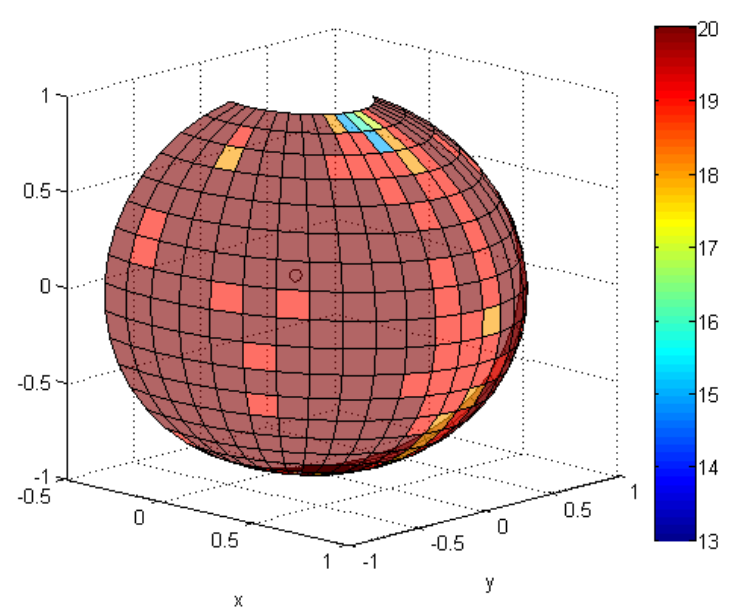

Fig. 3. Optimized static workspace. $(0,0,0)$ is shoulder center, positive $\mathrm{x}$ is forward, positive $\mathrm{y}$ is left. For each point of grid (plane of elevation, angle of elevation), shading represents number of internal/external rotation positions which are feasible out of 20 tested. Points are feasible if it is possible to find a nonnegative cable tensions to hold arm stationary at that point.

Due to the unique construction of the cable-driven exoskeleton, as compared to traditional rigid exoskeletons, extra considerations have to be given in terms of optimal cable attachment points to ensure a large feasible workspace for the arm. Unlike rigid links, cables can only be effective while in tension, so the location of the cables becomes very important.

Briefly, the cable positions were selected by optimizing the area of the static workspace [15]. The static workspace was calculated by setting $\ddot{\mathrm{q}}$ and $\dot{\mathrm{q}}$ equal to 0 in (1), and then determining the total number of feasible points where the arm can be in equilibrium under gravity. For each point, if there is a combination of non-negative cable tensions which satisfies (1), the point was considered feasible [16]. This process was repeated as the angular position of the shoulder cables was allowed to vary along both the shoulder and bicep cuff. The elbow cable positions were fixed by anatomical considerations, and the radial positions along the shoulder 
cuff were set to simplify the optimization. The final static workspace of the shoulder can be seen in Fig. 3. The mesh grid along the surface of a sphere (centered at $(0,0,0)$, the shoulder center) represents the tested points in polar coordinates of $\mathrm{q}_{1}, \mathrm{q}_{2}$. The shaded color represents the number of feasible $\mathrm{q}_{3}$ positions tested, out of a total of 20 .

\section{Computational Structure}

Again, due to the requirement of non-negative tension values in the cables, the control architecture is a bit more complicated than simply equating a desired control law with the equation of motion. However, to solve the system, we can take advantage of a unique property of the Jacobian. The Jacobian takes the form of a $6 \times 4$ matrix, since there are 4 degrees of freedom and 6 cables in the system. This can be written as

$$
\mathrm{AT}=\mathrm{B} \text {, }
$$

where $A$ is $J(q)^{T}$, T is the vector of cable tensions, and $B$ is the left side of the equations of motion, eqn. (1). It is important to note that the tension in the four cables that terminate at the bicep cuff have no direct effect on the elbow angle, so the Jacobian has a special structure, as shown in the next equation:

$$
A=\left[\begin{array}{cccc} 
& A_{1}^{3 \times 4} & & A_{2}^{3 \times 2} \\
& 1 & & A_{3}^{1 \times 2}
\end{array}\right] .
$$

Due to this special structure, it is possible to decompose the problem into analyzing the shoulder and elbow cases separately. On solving for the elbow first, independent of the shoulder, it simplifies the computation of a feasible solution by decreasing the number of equations to be solved simultaneously. The elbow component then takes the form

$$
\mathrm{A}_{3} \mathrm{~T}(5: 6)=\mathrm{B}(4)
$$

Here, $\mathrm{T}(5: 6)$ represents the tension vector in the last two cables and $\mathrm{B}(4)$ represents the $4^{\text {th }}$ element of the vector $\mathrm{B}$. The desired control law is used to find $\mathrm{T}(5: 6)$. If the resulting tension specified by the control law is negative, it cannot be applied to the exoskeleton, since cables can only 'pull' but not 'push'. Instead, if the solution of the controller is negative, the solution can be written as

$$
\mathrm{T}=\overline{\mathrm{T}}+\mathrm{N}(\mathrm{A}) \mathrm{m}
$$

where $\overline{\mathrm{T}}$ is a minimum norm solution provided by

$$
\overline{\mathrm{T}}=\mathrm{A}^{\mathrm{T}}\left(\mathrm{AA}^{\mathrm{T}}\right)^{-1}[\mathrm{C}] \text {, }
$$

where $N(A)$ is the nullspace of $A, m$ is a variable used to modify the tensions, and $\mathrm{C}$ is determined by the choice of a specific control law [16]. If no value of $m$ can be chosen such that the tensions are all non-negative, those tensions which are negative are set to zero. Theoretically, the system should then be able to compensate for this infeasibility at the next iteration, as most points do have feasible solutions, assuming that the errors will not propagate.

Once the elbow tension has been solved, the computed elbow cable tensions $\mathrm{T}(5: 6)$ can then be used to modify (2) and calculate the shoulder cable tensions according to

$$
\mathrm{A}_{1} \mathrm{~T}(1: 4)=\mathrm{B}(1: 3)-\mathrm{A}_{2} \mathrm{~T}(5: 6)
$$

where $\mathrm{T}(1: 4)$ are the first 4 elements of the tension vector, and $\mathrm{B}(1: 3)$ are the first three elements of the B vector. From here, the shoulder is solved in the same manner as was the elbow, since the entire right side of the (7) is known.

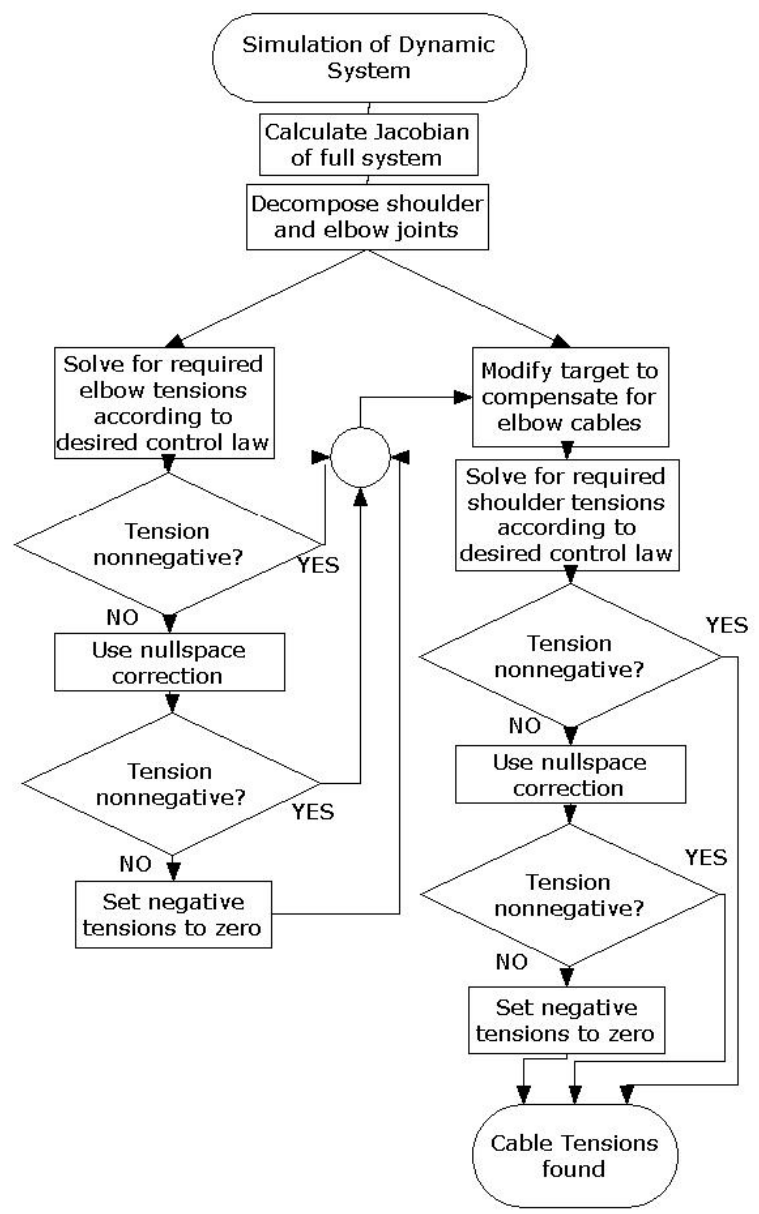

Fig. 4: Flowchart of controller architecture. System is separated into shoulder and elbow components; elbow is solved first, and then shoulder is solved with compensation for elbow result.

\section{E. Simulation}

Simulations were used to determine the effectiveness of the controllers on the exoskeleton. Simulations were done in Matlab using the ode solver ode15s for the differential equations of motion.

\section{CONTRoller InVEStigations}

\section{A. Set-point Controller}

A set-point controller was implemented first on the arm exoskeleton to determine how well the reachable dynamic workspace corresponded to the static feasible region previously calculated. This controller was a simple way to investigate the dynamic workspace. The controller was a modified PD set point control. The equation of motion was rewritten as: 


$$
\mathrm{D}(\mathrm{q}) \ddot{\mathrm{q}}+\mathrm{C}(\mathrm{q}, \dot{\mathrm{q}}) \dot{\mathrm{q}}+\mathrm{g}(\mathrm{q})=\mathrm{u}
$$

so that the control law could then be written as:

$$
\mathrm{u}=\mathrm{K}_{\mathrm{P}}\left(\mathrm{q}_{\mathrm{d}}-\mathrm{q}\right)-\mathrm{K}_{\mathrm{D}} \dot{\mathrm{q}}+\mathrm{g}(\mathrm{q})
$$

where the $\mathrm{g}(\mathrm{q})$ term represents the gravitational torque, which is added to remove steady state error, $\mathrm{K}_{\mathrm{P}}$ is the proportional gain term, $\mathrm{K}_{\mathrm{D}}$ is the derivative gain, and $\mathrm{q}_{\mathrm{d}}$ is the target point. The gain values were manually adjusted to improve the performance of the controller. The following gain values were computed to be best based on the simulation parameters: $\mathrm{K}_{\mathrm{P}}=70$ and $\mathrm{K}_{\mathrm{D}}=40$.

First, the PD set-point controller was tested with a set-point that was identical to the starting position, to ensure that the controller could maintain static stability within the static feasible area previously determined. This worked quite well in most cases, and supported the stability and functionality of the control architecture.
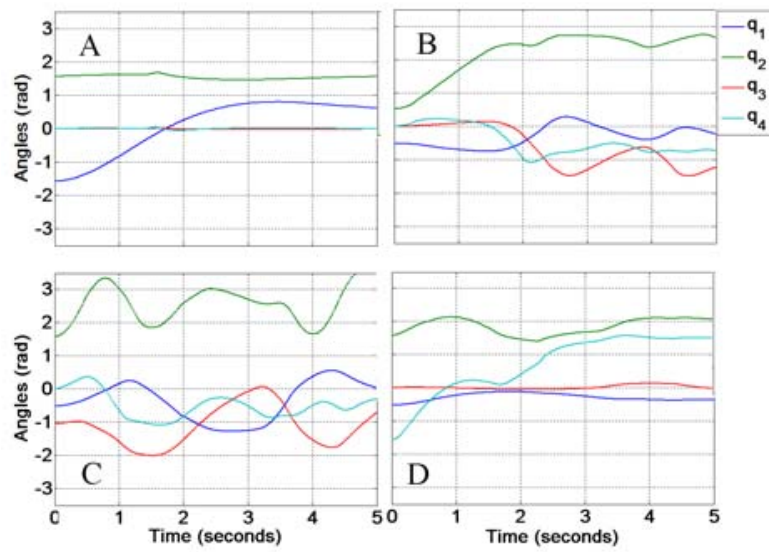

Fig. 5. Simulation results showing position of all joints. Desired motion is in only one joint at a time using PD set-point controller: A) $\mathrm{q}_{1}$ motion, B) $\mathrm{q}_{2}$ motion, C) $\mathrm{q}_{3}$ motion, D) $\mathrm{q}_{4}$ motion. For each plot, all other joint angles should remain constant. The joints are unable to move entirely independently of one another.

Next, the PD set-point controller was used to broadly investigate the range of the dynamic workspace by attempting to move the arm through a full range of motion in one single degree-of-freedom, while simultaneously keeping the others constant. The results of these simulations are displayed in Fig. 5. When angles $\mathrm{q}_{1}$ or $\mathrm{q}_{4}$ are in motion, the other angles remain fairly constant. However, when $\mathrm{q}_{2}$ or $\mathrm{q}_{3}$ are in motion, the other angles do not remain constant, but are instead affected by the motion in those joints.

While the controller is able to attempt the desired motion and may in some cases approach it, it is not possible to move exactly to a point at all places within the workspace. This is due to the dynamic coupling of the actuators to multiple joint motions. Also, in some cases, the required torque to carry out the dynamic motion may not be decomposed into positive cable tensions. At these infeasible points, the resulting tension is adjusted to the best the system can apply, but is not sufficient to maintain the desired position.

The reason it is not possible to move to all places within the static workspace, is that although the optimized static workspace shows the shoulders range of feasibility, it does not consider the dynamic effects of the cables that control the elbow. When the elbow is included, a tension is required in one of these cables; this tension then also acts on the bicep cuff, causing motion at the shoulder joint, and changing the required tensions of the shoulder cables to maintain the static equilibrium.

Additionally, in select cases, if the elbow is flexed past anatomical 90-degrees, the elbow extension cable will pass anterior to the elbow joint, meaning that both cables can provide only a flexion torque. The control algorithm bypasses this problem by applying no tension to the elbow cables, but this perturbs the system away from the target.

\section{B. Computed Torque Controller}

To attempt to address the shortcomings of the PD set-point controller, a computed torque controller was implemented, since it can better utilize the dynamics of the model than the PD controller. The control law was written as:

$$
\mathrm{u}=\mathrm{M}(\mathrm{q})\left(\ddot{\mathrm{q}}_{\mathrm{d}}-\mathrm{K}_{1} \dot{\mathrm{e}}-\mathrm{K}_{0} \mathrm{e}\right)+\mathrm{h}(\mathrm{q}, \dot{\mathrm{q}})
$$

where $\mathrm{e}$ is calculated as actual position minus the desired position, $\mathrm{M}(\mathrm{q})$ is the mass matrix, and $\mathrm{h}(\mathrm{q}, \dot{\mathrm{q}})$ includes the Coriolis and potential due to gravity terms, and $\mathrm{K}_{0}$ and $\mathrm{K}_{1}$ are the gain values. Like the PD set-point controller, the computed torque controller was equally able to maintain a steady state position, supporting the stability of this controller as well. When given a slowly changing sinusoidal trajectory to follow, however, the computer torque controller is still not able to maintain the desired trajectory.

\section{Trajectory Tracking PD Controller}

Finally, the original PD set-point controller was used in a different way so that it was able to follow a series of individual points. This has the advantage of being able to prescribe the exact set of points through which to move. Though this could also have been done with the computed torque controller, the performance of the PD controller seemed more stable, and it was used instead. In this case, the control law remained the same, but the task was slightly different. It was thought that perhaps given a series of points through which to move, the error of the PD controller could be minimized. Additionally, the ability to follow a series of set-points would allow the device to be tested with standardized trajectories of joint motion during activities of daily living, such as presented by van Andel et al. [17]. First, however, a series of only 4 set-points was tested, with each point held for 1 second. The controller is able to follow fairly well for the first three seconds, but then it veers significantly off the desired trajectory.

\section{EXPERIMENT}

\section{A. Experiment setup}

A photograph of the experiment setup is shown in Fig. 6 . The cable driven arm exoskeleton is mounted on an 
off-the-shelf plastic skeleton. The six motors, shoulder cuff, arm cuffs, and cable reels are mounted to get optimized workspace [15]. The motor amplifiers provide closed-loop speed control and encoders are mounted on the joints within the plastic skeleton. A schematic of the implementation architecture is shown in Fig. 7.
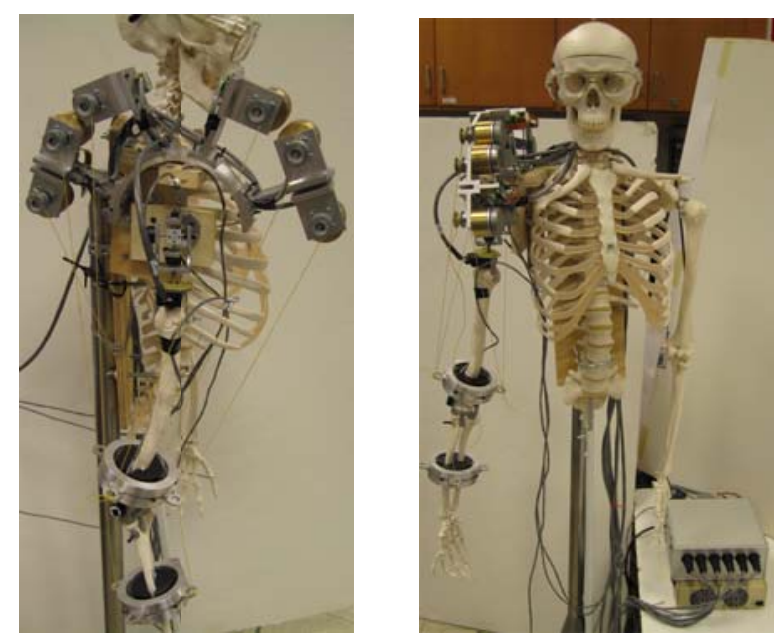

Fig. 6. The skeleton mounted with the cable driven arm exoskeleton viewed from side and the front.

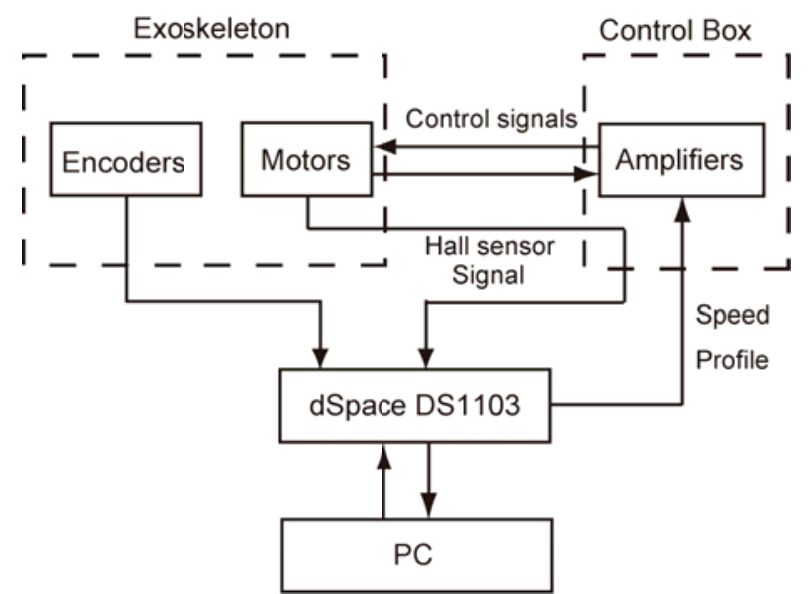

Fig. 7. Schematic of the experiment implementation architecture.

\section{B. Experiment results}

Fig. 8 shows a representative speed profile for the motors supplied to the experiment and the actual speed profile measured from experiment. The cable rate data was used to compute the joint angles of the plastic skeleton theoretically and was compared to experimentally measured joint data in Fig. 9. We want to emphasize that these experiments are only preliminary and we are investigating other variations of control architectures which rely on tension based control.

\section{CONCLUSIONS AND Future Work}

It was found that the standard PD controllers or Computed torque controllers, commonly used in the field of robotics, have only a moderate chance of success in controlling the cable-driven wearable arm exoskeleton. While the static feasible workspace of the exoskeleton seemed to suggest that the PD and computed torque controllers should be able to adequately control the exoskeleton within the range, it appears that the inertial and nonlinear dynamic coupling terms cause tracking errors, due to lack of positivity of tensions. In future, we will actively investigate other control architectures which ensure positivity of cable tensions, while still assuring point-to-point motion of the system.
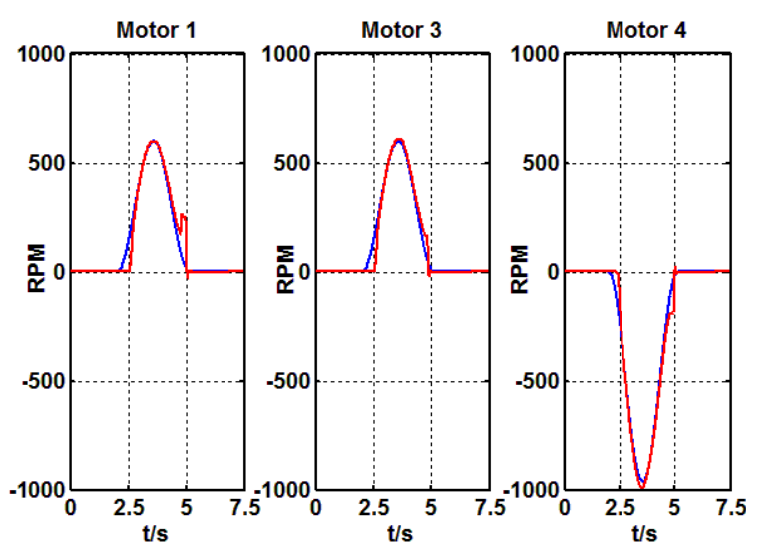

Fig. 8. Prescribed speed profile for Motor 1,3,4. Other motors' speeds are set to 0 in this experiment.
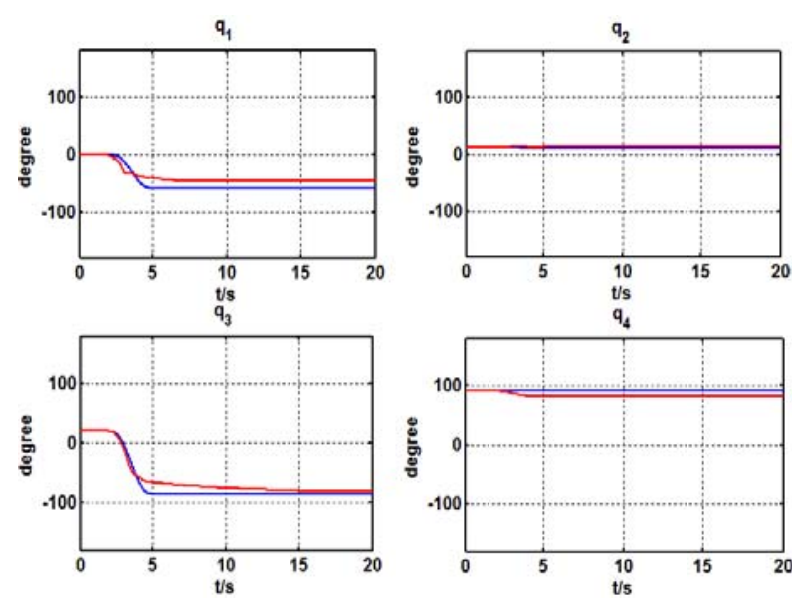

Fig. 9. Joint angle trajectory when motor speed profiles in Fig.10 are fed to the system. Blue line: calculated trajectory. Red line: experiment trajectory.

\section{ACKNOWLEDGMENT}

We would like to thank John Gangloff for design and fabrication assistance, and Vivek Sangwan for help with the dynamics. The third author gratefully acknowledges funding from WCU program.

\section{REFERENCES}

[1] L. Lunenburger, G. Colombo, R. Riener and V. Dietz, "Biofeedback in gait training with the robotic orthosis 
lokomat," in 26th Annual International Conference of the IEEE, 2004, pp. 4888-4891.

[2] A. Chu, H. Kazerooni and A. Zoss, "On the biomimetic design og the berkeley lower extremity exoskeleton (BLEEX)," in Proceedings of the 2005 IEEE ICRA, 2005, pp. 4345-4352.

[3] T. Hayashi, H. Kawamoto and Y. Sankai, "Control method of robotic suit HAL working as operator's muscle using biological and dynamical information," in 2005 IEEE/RSJ IROS, 2005, pp. 3063-3068.

[4] C. Carignan, M. Liszka and S. Roderick, "Design of an arm exoskeleton with scapula motion for shoulder rehabilitation," Advanced Robotics, 2005. ICAR '05. Proceedings., 12th International Conference on, pp. 524-531, 2005.

[5] A. Gupta and M. K. O'Malley, "Design of a haptic arm exoskeleton for training and rehabilitation," Mechatronics, IEEE/ASME Transactions on, vol. 11, pp. 280-289, 2006.

[6] T. Nef, M. Mihelj, G. Kiefer, C. Perndl, R. Muller and R. Riener, "ARMin - Exoskeleton for Arm Therapy in Stroke Patients," Rehabilitation Robotics, 2007. ICORR 2007. IEEE 10th International Conference on, pp. 68-74, 2007.

[7] K. Kiguchi and et al, "Development of a 3dof mobile exoskeleton robot for human upper-limb motion assist," Robot Auton Syst, In Press.

[8] J. C. Perry, J. Rosen and S. Burns, "Upper-Limb Powered Exoskeleton Design," Mechatronics, IEEE/ASME Transactions on, vol. 12, pp. 408-417, 2007.

[9] Zheng Man, Weihai Chen, Shouqian Yu and Xingming $\mathrm{Wu}$, "Kinematic Analysis for a 7-DOF Modular Hybrid-Driven Manipulator," Industrial Informatics, 2006 IEEE International Conference on, pp. 1363-1368, 2006.

[10] G. Yang, M. S. Kurbanhusen, C. B. Pham and S. H. Yeo, "Kinematic design of a 7-dof cable-driven humanoid arm:a solution-in-nature approach," in Proceedings of the 2005 IEEE/ASME International Conference on Advanced Intelligent Mechatronics, 2005, pp. 444-449.

[11] S. K. Agrawal and A. Fattah, "Design of an orthotic device for full or partial gravity-balancing of a human upper arm during motion," in Proceedings of the 2003 IEEE/RSJ IROS, 2003, pp. 2841-2846.

[12] S. K. Banala, S. K. Agrawal, A. Fattah, V. Krishnamoorthy, W. Hsu, J. Scholz and K. Rudolph, "Gravity-balancing leg orthosis and its performance evaluation," IEEE Trans. Robot., vol. 22, pp. 1228-1239, Dec 2006. 2006.

[13] T. Rahman, W. Sample, R. Seliktar, M. T. Scavina, A. L. Clark, K. Moran and M. A. Alexander, "Design and Testing of a Functional Arm Orthosis in Patients With Neuromuscular Diseases," Neural Systems and Rehabilitation Engineering, IEEE Transactions on, vol. 15, pp. 244-251, 2007.

[14] G. Wu and et al., "ISB recommendation on definitions of joint coordinate systems of various joints for the reporting of human joint motion-part II: shoulder, elbow, wrist, hand," J Biomech, vol. 38, pp. 981-992, 2005.

[15] S. K. Agrawal, V. N. Dubey, J. Gangloff, E. Brackbill and V. Sangwan, "Optimization and Design of a cable driven upper arm exoskeleton," under review for Journal of Medical Devices, Transactions of the ASME, 2008.

[16] S. Oh and S. K. Agrawal, "Cable suspended planar robots with redundant cables: controllers with positive tensions," IEEE Transactions on Robotics, vol. 21, pp. 457-464, 2005.

[17] C. J. Van Andel, N. Wolterbeek, C. A. Doorenbosch, D. H. Veeger and J. Harlaar, "Complete 3D kinematics of upper extremity functional tasks," Gait Posture, vol. 27, pp. 120-127, Jan. 2008. 